\title{
Teil C: Relevanz von Arzt- und Arbeitsrecht
}

C.1. Die Bedeutung des Arztrechts für die BMB

C.2. Die Bedeutung des Arbeitsrechts für BMB 



\section{C.1. Die Bedeutung des Arztrechts für die BMB}

Das Arztrecht ist ein Teilrechtsgebiet des so genannten Gesundheitsrechts. Unter Arztrecht versteht man sämtliche zivil- und öffentlich-rechtlichen Normen, die das Recht der Berufszulassung und der Berufsausübung der Ärzte regeln. Von besonderer Bedeutung sind dabei die rechtlichen Rahmenbedingungen, unter denen der Arzt die Heilbehandlung an seinen Patienten ausübt. Zivilrechtliche Grundlage für das Rechtsverhältnis zwischen Arzt und Patient ist regelmäßig der Behandlungsvertrag. Das Rechtsverhältnis zwischen Arzt und Patient wird mithin einerseits durch dieses Vertragsverhältnis andererseits durch zwingende gesetzliche Regelungen bestimmt.

In den folgenden Betrachtungen zur Relevanz des Arztrechts für BMB wird dahingehend unterschieden, ob die in die BMB einzustellenden Körpermaterialien im Rahmen eines Behandlungs- bzw. Diagnosevertrages oder ausschließlich zu Forschungszwecken, d.h. ohne medizinische Indikation gewonnen werden. Es ist jedoch schon hier darauf hinzuweisen, dass nach \23 MBO-Ä I997 alle im Folgenden diskutierten berufsrechtlichen Pflichten eines Arztes von diesem unabhängig davon zu beachten sind, ob er seinen Beruf selbständig oder in einem Anstellungsverhältnis ausübt. Ein Arzt darf nach \2 Abs. 4 MBO-Ä I997 hinsichtlich seiner ärztlichen Entscheidungen keine Weisungen von Nichtärzten entgegennehmen.

\section{C.1.1. Probengewinnung im Rahmen eines Behandlungs-/ Diagnosevertrages}

Sofern der Patient aufgrund einer medizinischen Indikation einen Arzt oder ein Krankenhaus aufsucht, um sich dort einer Heilbehandlung zu unterziehen, wird zwischen ihm und dem Arzt oder Krankenhaus ein Behandlungsvertrag geschlossen. Hierbei handelt es sich, wie unter B.I.4.2.I. bereits ausgeführt, um einen Dienstvertrag nach $\int$ 6II BGB. Der Arzt schuldet dem Patienten als Hauptleistungspflicht das Bemühen um die Behandlung der Erkrankung nach den Regeln der ärztlichen Kunst, d.h. eine Behandlung auf Facharztniveau. ${ }^{90}$ Er hat für den Erfolg der Behandlung regelmäßig jedoch nicht einzustehen. ${ }^{91}$ Von dem Moment an, wo sich dieses Vertragsverhältnis zwischen Arzt und Patient anbahnt, kommt grundsätzlich das gesamte Arztrecht zur Anwendung. Von besonderer Bedeutung sind dabei die Vorschriften des BGB, das ärztliche Berufsrecht, welches im Wesentlichen in der Bundesärzteordnung, der Approbationsordnung und der Muster-Berufsordnung für die deutschen Ärztinnen und Ärzte ${ }^{92}$ (MBO-Ä I997) besteht, ferner das Transfusionsgesetz (TFG), das Transplantationsgesetz (TPG) sowie die einschlägigen Vorschriften des StGB über die Körperverletzung

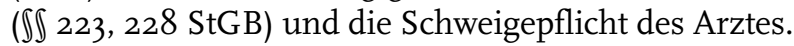

90 Dettmeyer (2001), Medizin \& Recht für Ärzte, S. 12.

91 Nur ausnahmsweise wird ein Werkvertrag angenommen, so dass auch ein Erfolg geschuldet wird (z.B. beim Anpassen einer Prothese).

92 Einige Länder haben eigene Landesberufsordnungen für Ärzte, die aber im Wesentlichen der MBO-Ä entsprechen. 


\section{C.1.1.1. Gewinnung der Körpermaterialien}

Die Gewinnung von Körpermaterialien im Rahmen eines Behandlungs- oder Diagnosevertrages kann unter verschiedenen Umständen erfolgen. Zunächst einmal ist es möglich, dass der Arzt dem Patienten zu Diagnosezwecken Körpermaterialien entnehmen muss (z.B. Blut, Gewebe oder Körperflüssigkeiten). Daneben kann im Rahmen einer Operation Körperteile (Amputation), Organe (Blinddarm, Niere) oder Gewebe (z.B. Tumoren) entnommen werden. In bestimmten Fällen ist auch denkbar, dass Körpermaterial als „Abfallprodukt“ im Rahmen des medizinischen Eingriffs anfällt (z.B. die Plazenta und Nabelschnurreste nach einer Geburt, Blut- und Gewebeteile nach einer Abtreibung, Knorpelmasse bei einem Oberschenkelhalsbruch, austretender Liquor oder Blut bei offenen Verletzungen). In allen diesen Situationen kommt es nur deshalb zum Anfall von Körpermaterialien, weil zwischen Arzt und Patient ein Behandlungsvertrag geschlossen wurde, aufgrund dessen der Arzt Zugriff auf das Körpermaterial hatte.

\section{C.1.1.2. Arztvorbehalt}

Alle unter C.I.I.I. genannten Eingriffe stellen Tätigkeiten der Heilkunde dar und stehen daher grundsätzlich unter Arztvorbehalt. Diese Eingriffe darf in der Regel nur ein Arzt durchführen; anderen Personen ist die Ausübung der „berufsmäßigen Heilkunde“ nach 』 I Heilpraktikergesetz nur mit Erlaubnis möglich. Ein Arzt darf einzelne der genannten Eingriffe wie z.B. die Blutentnahme ${ }^{93}$ nur ausnahmsweise auf hinreichend qualifiziertes nichtärztliches Personal delegieren. Voraussetzung hierfür ist allerdings, dass bei der Ausführung des Eingriffs der Standard eines erfahrenen Facharztes gewährleistet ist. ${ }^{94}$ Folgende ärztliche Aufgaben sind indes unter keinen Umständen delegierbar:

- Operative und endoskopische Eingriffe

- Ärztliche Untersuchung und Beratung des Patienten

- Aufklärungsgespräche.

Hierbei handelt es sich um Hauptleistungspflichten des Arztes aus dem Behandlungsvertrag, die in der Regel höchstpersönlich zu erbringen sind, mithin sogar nur eingeschränkt auf einen anderen Arzt übertragen werden dürfen.

\section{C.1.1.3. Einwilligungsvorbehalt}

Jeder ärztliche Eingriff in den Körper eines Patienten bedarf dessen vorheriger Einwilligung, andernfalls stellt der Eingriff eine Körperverletzung im Sinne der

93 In der Stellungnahme der Arbeitsgemeinschaft Deutscher Schwesternverbände und des Deutschen Berufsverbandes für Krankenpflege e.V. zur Vornahme von Injektionen, Infusionen, Transfusionen und Blutentnahmen (Stellungnahme Injektionen) vom April 1989 wird ausdrücklich festgestellt, dass die Blutentnahme in das Gebiet der Heilkunde fällt und damit in erster Linie zum Aufgaben- und Verantwortungsbereich der Ärzte gehört.

94 Reinhard Dettmeyer (2001), Medizin und Recht für Ärzte, S. 286; vgl. auch den Beschluss des Vorstandes der Bundesärztekammer vom 18.04.1980 (Deutsches Ärzteblatt vom 03.07.1980). 
$\iint 223$ f. StGB dar. Dieser Grundsatz findet sich auch in $\int 8$ MBO-Ä wieder, wonach jede Behandlung der Einwilligung des Patienten bedarf. Zudem bestimmt $\int 7$ MBO-Ä, dass jede medizinische Behandlung unter Wahrung der Menschenwürde und der Achtung der Persönlichkeit, des Willens und der Rechte des Patienten zu erfolgen hat.

Beabsichtigt der Arzt, Körpermaterial zu Zwecken zu entnehmen, die nicht unmittelbar der Heilbehandlung des Patienten dienen, muss er den Patienten darüber aufklären und die Einwilligung des Patienten in diese Entnahme einholen. Die Einwilligung des Patienten in die Heilbehandlung als solche genügt nicht. ${ }^{95}$ Selbst wenn die Untersuchung zu einem für den Patienten bedeutungsvollen Ergebnis führt, gilt die durchgeführte Entnahme als nicht erlaubt, wenn hierzu keine Einwilligung vorlag. ${ }^{96}$

Es ist allgemein anerkannt, dass selbst die medizinische Indikation für eine bestimmte Untersuchungs-, Behandlungs- oder Diagnosemaßnahme die Einwilligung des Patienten in diese Maßnahme nicht ersetzen kann. ${ }^{97}$ Eine heimlich durchgeführte Maßnahme verletzt das allgemeine Persönlichkeitsrecht des Patienten..$^{98} \mathrm{Da}$ das allgemeine Persönlichkeitsrecht ein sonstiges Recht im Sinne des $\int 823$ I BGB darstellt, begründet die Verletzung des allgemeinen Persönlichkeitsrechts einen Anspruch auf billige Entschädigung in Geld, mithin auf Schmerzensgeld. ${ }^{99}$ Weder die Forschungsfreiheit noch das erstrebte Ergebnis können einen heimlich durchgeführten Eingriff rechtfertigen. ${ }^{100}$ In diesem Zusammenhang wird auch auf die öffentlich vertretene Ansicht einer Ethikkommission verwiesen, dass selbst bei einer zeitlich wesentlich späteren Untersuchung von Organen, die im Rahmen einer Sektion einem Toten entnommen wurde, dies nur mit Einwilligung der Hinterbliebenen erlaubt sei. Auch bei einer vorherigen Anonymisierung würde weiterhin das nachwirkende Persönlichkeitsrecht des Verstorbenen verletzt werden, wenn die Einwilligung nicht eingeholt wird. ${ }^{101}$

\section{C.1.1.4. Aufklärungspflicht}

Der Einwilligung des Patienten in die Entnahme von Körpermaterialien hat grundsätzlich eine Aufklärung über den Eingriff vorauszugehen. Diese Aufklärung muss sich auf den medizinischen Eingriff als solchen und auf sämtliche, damit verbundene Risiken beziehen. Die Aufklärung soll dazu führen, dass der Patient Art, Bedeutung, Dringlichkeit, Verlauf und Folgen des Eingriffs zumindest in Grundzügen versteht. Da die Entnahme von Körpermaterial grundsätzlich eine ärztliche Maßnahme ist, kann die Aufklärung über Zweck und Folgen

95 Deutsch et al., Transfusionsrecht, Rdnr. 469 (465 f.), der aber die Ansicht vertritt, dass die Entnahme als solches nicht rechtswidrig wird, da sie von der Einwilligung gedeckt ist. Nur die Weiterverwendung sei rechtswidrig und würde eine Schadenersatzpflicht begründen.

96 LG Köln, in: MedR 1995, 409; Laufs (1993), Arztrecht, München 1993, Rdnr. 230.

97 LG Köln, in: MedR 1995, 409.

98 Nach Laufs (1993), Rdnr. 230, sog. Ausforschungsdiagnostik.

99 Vgl. Deutsch et al., Transfusionsrecht, Rdnr. 471 m.w.Nw.

100 Laufs (1993), Rdnr. 230.

101 Dettmeyer (2001), S. 276. 
des damit verbundenen Eingriffs nur durch einen Arzt erfolgen und kann nicht auf nicht-ärztliches Personal delegiert werden

Die Aufklärung hat so rechtzeitig zu erfolgen, dass der Patient frei entscheiden kann, ob er der Durchführung des Eingriffes zustimmt. Etwas anderes kann nur ausnahmsweise bei besonderer Dringlichkeit gelten. Der Inhalt der Aufklärung muss sich auf alle Aspekte des Eingriffs beziehen, die bei einem medizinischen Laien als nicht hinreichend bekannt vorausgesetzt werden müssen (als Allgemeinwissen, und damit als hinreichend bekannt, gilt z.B. die Möglichkeit einer Hämatombildung bei der Blutentnahme) ${ }^{102}$ Der Patient muss über mögliche Komplikationen, über den Verlauf der Maßnahme und über die Folgen eines Unterlassens der Maßnahme aufgeklärt werden. Er soll zudem über Diagnosen, Prognosen, Therapie, Ziele der Behandlung, deren Notwendigkeit und Dringlichkeit aufgeklärt werden. Dies alles ist notwendig, weil es dem Patienten aufgrund seines Selbstbestimmungsrechtes als Ausfluss des allgemeinen Persönlichkeitsrechtes frei steht, selbst dringend notwendige Eingriffe zu unterlassen..$^{103}$

Eine nicht oder nicht im erforderlichen Umfang durchgeführte Aufklärung eines Patienten führt dazu, dass die Einwilligung als nicht erteilt gilt. Dies führt neben einem deliktsrechtlichen Anspruch des Patienten gegen den behandelnden Arzt (siehe C.I.I.3.) auch dazu, dass der Eingriff eine Körperverletzung im Sinne des $₫ 223$ StGB darstellt. ${ }^{104}$

\section{C.1.1.5. Weiternutzung des Körpermaterials zu Forschungszwecken}

Unabhängig von Art und Umfang der Nutzung des Körpermaterials wird der Vorgang der Entnahme zum Zeitpunkt der Nutzung im Allgemeinen bereits abgeschlossen sein. Die Entnahme als solches bleibt daher von der Einwilligung des Patienten gedeckt und wird in keinem Fall rückwirkend rechtswidrig. ${ }^{105} \mathrm{Al}-$ lerdings stellt eine unerlaubte Weiterverwendung des Körpermaterials nach Ansicht der Gutachter eine Verletzung des Behandlungsvertrages dar, denn sie ist dem Arzt nicht erlaubt worden.

Wie wir unter B.I.2. festgestellt haben, werden Körpermaterialien, die endgültig vom Körper eines Menschen getrennt wurden und nicht wieder in diesen eingegliedert werden sollen, Sachen im Sinne des BGB. Ohne ausdrückliche Übereignungsvereinbarung stehen die Materialien daher weiterhin im Eigentum des Patienten. Die Beforschung der Materialien ohne Einwilligung des Patienten stellt einen unberechtigten Eingriff in das Sacheigentum dar und kann somit den Tatbestand einer Unterschlagung im Sinne des $\ 246$ StGB oder einer Sachbeschädigung im Sinne des $₫ 303$ StGB erfüllen. Zudem dürfte das allgemeine Persönlichkeitsrecht des Patienten verletzt sein, welches die Sacheigenschaft der Materialien nach herrschender Ansicht überlagert (siehe

102 BGH NJW 1992, 743; Dettmeyer (2001), S. 31.

103 Dettmeyer (2001), S. 30.

104 Dettmeyer (2001), S. 27.

105 Deutsch, et al., Transfusionsrecht, Rdnr. 469 (465 f.). 
B.I.3.I.). ${ }^{106}$ In jüngster Zeit mehren sich daher Stimmen, die dem Patienten als besondere Ausprägung des allgemeinen Persönlichkeitsrechts ein so genanntes „Recht auf biomaterielle Selbstbestimmung“ zugestehen, d.h. selbst zu entscheiden, wie mit seinen Körpermaterialien zu verfahren ist. ${ }^{107}$

Jeder Patient hat das alleinige Recht darüber zu entscheiden, was mit seinen Körpermaterialien geschieht. Wenn er hierzu keine ausdrückliche Erklärung abgegeben hat (z.B. in Form einer Übereignungsvereinbarung), so ist das Körpermaterial zu vernichten, sofern es nicht aus medizinischen Gründen per Nebenpflicht aus dem Behandlungsvertrag oder aufgrund einschlägiger Dokumentationsvorschriften aufbewahrt werden muss (siehe B.I.4.2.I.a.). Die nicht von der Einwilligung des Patienten gedeckte Weiternutzung von Körpermaterialien, die im Rahmen eines ärztlichen Eingriffs anfallen, verletzt das allgemeine Persönlichkeitsrecht und das Eigentumsrecht des Patienten. ${ }^{108}$ Der Arzt würde damit nach Ansicht der Gutachter sowohl gegen $\ 7$ MBO-Ä als auch gegen $\$ II MBO-Ä verstoßen. Sein Verhalten stellt zudem einen Diebstahl gemäß \242 StGB dar. Hat sich der Patient dem entgegen mit dem Eigentumsübergang auf die BMB bzw. die entnehmende Stelle einverstanden erklärt, ist die Weiternutzung der Körpermaterialien zu Forschungszwecken nach Maßgabe der berufsrechtlichen Vorschriften grundsätzlich erlaubt.

\section{C.1.1.6. Pflicht zur Einschaltung der Ethikkommission}

Für jede biomedizinische Forschung am Menschen oder Forschung mit vitalen menschlichen Gameten oder lebendem embryonalem Gewebe muss sich ein Arzt nach 』I5 MBO-Ä durch eine Ethikkommission beraten lassen. Eine Ausnahme gilt nach $\int I_{5}$ Abs. I MBO-Ä nur für die epidemiologische Forschung. Aufgabe der Ethikkommission ist es, die rechtliche und ethische Unbedenklichkeit des Forschungsvorhabens fest- und sicherzustellen. Dabei wird sie die Einhaltung sämtlicher strafrechtlich (z.B. \226 a StGB), zivilrechtlich (BGB: Einwilligungsvorbehalt, Eigentumsrechte) und öffentlich-rechtlich (GG, AMG, MBO-Ä, die Verordnung über die Anwendung der guten klinischen Praxis etc.) relevanten Vorschriften überwachen. In ethischer Hinsicht steht die Einhaltung der allgemeingültigen Voraussetzungen für wissenschaftliche Humanversuche (Revidierte Deklaration des Weltärztebundes von Helsinki, etc.) im Zentrum des Interesses.

Folgt man z.B. der Definition der Ethik-Kommission der Universität Göttingen, so bedeutet Forschung am Menschen

„jede, die somatische oder psychische Integrität eines Menschen berührende Maßnahme mit dem Ziel, über den Einzelfall hinaus präventive, diagnostische, therapeutische, pathophysiologische oder physiologische Erkenntnisse zu gewinnen." 
Demgemäß ist Forschung an entnommenen Körpermaterialien immer dann als Forschung am Menschen anzusehen, wenn die entsprechenden Materialien ausschließlich zum Zwecke der Forschung entnommen werden.

Bei der Forschung an Körpermaterialien, die im Rahmen eines medizinischen Eingriffs bzw. einer ärztlichen Heilbehandlung anfallen, erfolgt die Entnahme des Materials jedoch primär zum Wohle des Patienten, und der Forschungszweck wird meistens erst nach Abschluss der Behandlung definiert. Mithin erfolgt kein auf den Forschungszweck gerichteter Eingriff in die Integrität eines Menschen. Menschliche Körpermaterialien, die endgültig vom Körper des Menschen getrennt sind, fallen also nach Ansicht der Gutachter nicht in den Anwendungsbereich des $₫$ I5 MBO- ̈̈, wenn sie im Rahmen eines (aufgrund medizinischer Indikation und Einwilligung des Patienten rechtmäßigen) medizinischen Eingriffs als überschüssiges Material anfallen. Forschung an ihnen bedarf mithin nicht der Einwilligung einer Ethikkommission.

Diese Einschätzung lässt sich auch dahingehend stützen, dass vitale menschliche Gameten und lebendes embryonales Gewebe herrschender Meinung zufolge nicht als Sache, sondern (ähnlich wie eine Leiche) als res extra commercium, als „neue Gattung sui generis zwischen Person und Sache“, „Sonderform von Teil-, Vor- oder Zwischenpersonalität“ gelten. ${ }^{109}$ Dies ist Grundlage der expliziten Einbeziehung vitaler menschliche Gameten und lebenden embryonalen Gewebes in den Anwendungsbereich des $₫$ I5 II MBO-Ä, gleichwohl beide noch nicht den Charakter eines Menschen haben. Die entsprechende Regelung wäre allerdings nicht notwendig gewesen, wenn unter dem Begriff „Forschung am Menschen" auch die Forschung an menschlichen Körpermaterialien verstanden würde, die im Rahmen medizinischer Eingriffe anfallen. Vitale menschliche Gameten und lebendes embryonales Gewebe sind nämlich nach diesseitiger Ansicht durchaus als solche anzusehen. ${ }^{110}$

\section{C.1.1.7. Aufbewahrungs- und Dokumentationspflicht}

Gemäß \ Iо MBO-Ä hat ein Arzt über die in Ausübung seines Berufes gemachten Feststellungen und getroffenen Maßnahmen die erforderlichen Aufzeichnungen zu machen. Diese Aufzeichnungen dienen nach $₫$ IO Abs. I MBO-̈̈ ausdrücklich auch dem Interesse des Patienten. Ihre Anfertigung stellt somit eine Hauptpflicht aus dem Behandlungsvertrag dar. Nach Ansicht der Gutachter schließt diese Pflicht auch die Dokumentation des Verbleibs überschüssiger Körpermaterialien ein. Die Dokumentation ist nach \ Io Abs. 3 MBO-Ä für die Dauer von io Jahren nach Abschluss der Behandlung aufzubewahren, sofern sich aus anderen Vorschriften nicht sogar eine längere Aufbewahrungspflicht ergibt.

Wie bereits unter B.I.4.2.I.a. dargestellt, ist aus der Dokumentationspflicht jedoch keine Pflicht zur Aufbewahrung überschüssigen Körpermaterials abzu-

109 Vgl. Halàsz (2004), S. 42 f. mit weiteren Nachweisen und einem Überblick über sämtliche derzeit vertretene Positionen.

110 Wie hier Halàsz (2004), S.54 (42 f.). 
leiten, weder unmittelbar noch bei entsprechender Anwendung aus \Io MBO-Ä. Gleichwohl ist eine Pflicht zur Aufbewahrung der Körpermaterialien als Nebenpflicht zum Behandlungsvertrag immer dann anzunehmen, wenn dies aus medizinischen Gründen geboten ist. ${ }^{111}$ Liegen keine derartigen Gründe vor, ist der Arzt verpflichtet, die überschüssigen Körpermaterialien entweder an den Patienten herauszugeben oder zu vernichten, sofern der Patient nicht in der Lage ist, die Körpermaterialien sachgerecht aufzubewahren. ${ }^{112}$

\section{C.1.1.8. Datenschutz und ärztliche Schweigepflicht}

Der Arzt unterliegt wegen sämtlicher Tatsachen, die ihm in seiner Eigenschaft als Arzt anvertraut oder bekannt gegeben werden, der ärztlichen Schweigepflicht. Grundlage der Schweigepflicht ist das verfassungsrechtlich verankerte Recht auf informationelle Selbstbestimmung des Patienten. Zugleich wird die ärztliche Schweigepflicht in $\ 9 \mathrm{MBO}-\ddot{A}$ als Berufspflicht normiert und als Nebenpflicht aus dem Behandlungsvertrag angesehen. ${ }^{113}$ Ein Verstoß gegen diese Pflichten begründet eine Strafbarkeit nach \203 StGB. ${ }^{114}$ Zudem unterliegt der Arzt im Falle der Erhebung und Speicherung von Daten des Patienten den einschlägigen datenschutzrechtlichen Bestimmungen. Der \Io Abs. 5 MBO-Ä normiert insoweit ausdrücklich eine besondere Pflicht des Arztes, für Schutzund Sicherungsmaßnahmen zu sorgen, um die Veränderung, Vernichtung oder unrechtmäßige Verwendung zu verhindern. Daraus ergibt sich, dass die Weitergabe überschüssigen Körpermaterials ohne vorherige Anonymisierung zugleich auch ein Verstoß gegen die ärztliche Schweigepflicht und die einschlägigen datenschutzrechtlichen Bestimmungen darstellt. ${ }^{115}$

\section{C.1.1.9. Nachvertragliche Aufklärungs- und Informationspflicht}

Auch nach dem Ende einer medizinischen Behandlung ist der Arzt aus fortwirkendem Vertragsverhältnis verpflichtet, von sich aus alles zu tun, um die Schädigungen seines Patienten möglichst gering $\mathrm{zu}$ halten. ${ }^{116}$ Dies bedeutet, dass ihn eine nebenvertragliche Pflicht trifft, den Patienten über nicht erkannte Krankheiten aufzuklären. ${ }^{117}$ Wenn ein Arzt also an überschüssigen Körpermaterialien weitere, nicht vom Behandlungsvertrag umfasste Untersuchungen vor-

111 OLG Hamm, NJW-RR 2003, 807, 808.

112 Breyer (2004), in: MedR, 662.

113 Breyer (2004), in: MedR, 664.

114 Nur in Ausnahmefällen, wenn eine gesetzlich normierte Meldepflicht besteht (z. B. KrebsRegG etc.) oder für die Zukunft die Gefahr für ein Rechtsgut von hohem Wert droht (z. B. Gefahr für das Leben anderer Menschen), kann der Arzt unter dem Gesichtspunkt des rechtfertigenden Notstandes berechtigt sein, Patientengeheimnisse z.B. gegenüber der Polizei zu offenbaren (vgl. OLG Frankfurt a. M., NJW 2000, 875 ff.).

115 Breyer (2004), S. 664, hält eine Aufbewahrung in anonymisierter Form für zulässig; so auch Wellbrock (2003), in: MedR, S. 80 (77).

116 Deutsch/Spickhoff (2003), Medizinrecht, 4. Aufl. Berlin, Rdnr. 222.

117 Deutsch/Spickhoff (2003), Rdnr. 687. Am Konflikt zwischen Anonymisierungspflicht und nachvertraglicher Aufklärungspflicht sind bereits Prävalenzstudien zur Erfassung von Aidserkrankungen gescheitert; Laufs, Rdnr. 230, sieht ein anonymisiertes Verfahren bei einer Querschnittsstudie, das die Identifizierung des Trägers ausschließt, als unärztlich an. 
nimmt, und hierbei für den Patienten wesentliche Erkenntnisse über dessen Gesundheitszustand erfährt, so hat er das Recht und die Pflicht, den Patienten über diese Untersuchungsergebnisse aufzuklären. Dies gilt unbenommen der Tatsache, dass der Arzt wegen der möglicherweise unerlaubten Weiterverwendung des Körpermaterials, d.h. aufgrund einer Verletzung des allgemeinen Persönlichkeitsrechts des Patienten, schadenersatzpflichtig sein könnte.

\section{C.1.2. Probengewinnung ausschließlich zu Forschungszwecken}

Erfolgt die Gewinnung von Körpermaterialien im Rahmen eines Probandenvertrages ausschließlich zu Forschungszwecken, so wird regelmäßig kein Behandlungsvertrag vorliegen, d.h. Vertragsgegenstand ist kein medizinischer Heilerfolg. Beim Probandenvertrag handelt es sich regelmäßig um einen Vertragstypus eigener Art, der bezogen auf das Biomaterial Elemente eines Übereignungs- oder Nießbrauchvertrags verbunden mit kaufrechtlichen, schenkungsrechtlichen oder nutzungsrechtlichen Regelungen aufweist. Zudem muss dieser Vertrag eine datenschutzrechtliche und eine persönlichkeitsrechtliche Einwilligungsklausel enthalten. Obwohl originär kein Behandlungsvertrag vorliegt, können auch bei der Entnahme von Körpermaterialien zu Forschungszwecken arztrechtliche Aspekte zum Tragen kommen. Dies hängt davon ab, welche Art von Körpermaterialien auf welche Art und Weise gewonnen werden sollen.

\section{C.1.2.1. Arztvorbehalt}

Sofern für die Entnahme von Körpermaterialien kein körperlicher Eingriff notwendig ist, muss diese Entnahme auch nicht notwendig durch einen Arzt erfolgen. Dies gilt z.B. für die Gewinnung von Haarwurzeln, Fingernägeln, Hornhaut, Speichel oder Sperma. Demgegenüber unterliegt die Blutentnahme grundsätzlich dem Arztvorbehalt. Wie unter C.I.I.2. bereits ausgeführt, kann ein Arzt die Entnahme von Blut jedoch auf entsprechend ausgebildetes medizinisches Hilfspersonal (Arzthelferin, Krankenschwester etc.) übertragen. Der Arzt trägt dabei jedoch nach wie vor die Auswahlverantwortung. Die Entnahme von Gewebeproben wiederum ist regelmäßig mit einem medizinischen Eingriff verbunden, der nicht selten nur unter Lokalanästhesie durchgeführt werden kann. Ein solcher Eingriff stellt eine ärztliche Maßnahme dar, die nur Ärzten vorbehalten ist. Sie kann nicht auf medizinisches Hilfspersonal übertragen werden. Die Durchführung jeden Eingriffs hat nach denselben Grundsätzen zu erfolgen wie ein Eingriff zu Behandlungszwecken, was die notwendige Nachbehandlung bzw. -betreuung einschließt.

\section{C.1.2.2. Einwilligungsvorbehalt}

Unabhängig davon, ob die Gewinnung des Körpermaterials einem Arztvorbehalt unterliegt oder nicht, muss der Spender vorab seine Einwilligung in die 
Entnahme erklären. Auch das Abschneiden von Haaren stellt z.B. einen Eingriff in die körperliche Integrität dar, der unter Umständen so entstellend sein kann, dass dadurch sogar eine Körperverletzung möglich ist. Ein Eingriff in das allgemeine Persönlichkeitsrecht des Spenders findet dabei aber in jedem Fall statt, und in diesen muss der Spender vorab einwilligen. Ist ein ärztlicher Eingriff zur Materialentnahme erforderlich, so sind die Anforderungen an die Einwilligung des Spenders sogar noch strenger $\mathrm{zu}$ fassen. In diesem Fall findet das allgemeine Arztrecht Anwendung mit der Besonderheit, dass für die Entnahme zu Forschungszwecken keine medizinische Indikation vorliegt. In solchen Fällen wird auch vom „atypischen Arztvertrag“ gesprochen. Ein medizinischer Eingriff ohne Indikation aufgrund eines atypischen Arztvertrages stellt grundsätzlich eine Körperverletzung nach \223 StGB dar, die nach $\int 228$ StGB nur dann nicht rechtswidrig ist, wenn der Patient in den Eingriff eingewilligt hat und der Eingriff nicht sittenwidrig ist.

Ob ein Eingriff in den Körper eines Spenders sittenwidrig ist, hängt vom Einzelfall ab. Bejaht wird dies, wenn es zu dauerhaften Körperschäden des Spenders kommt (z. B. indikationslose Amputation). Auch die Organentnahme gegen Entgelt wird als sittenwidrig angesehen. Die indikationslose, möglicherweise entgeltliche und zugleich kommerzielle Entnahme von Körpermaterialien stellt nach Ansicht der Gutachter jedoch kein sittenwidriges Verhalten dar. Zum einen gibt es weder auf nationaler noch auf internationaler Ebene eine Regelung, die den entgeltlichen, kommerziellen Handel mit menschlichen Körpermaterialien untersagt, so dass daraus dessen Zulässigkeit abgeleitet werden kann. Zum anderen ist der Spender aufgrund seines grundrechtlich geschützten Selbstbestimmungsrechts uneingeschränkt berechtigt, einer Entnahme von Körpermaterialien zuzustimmen, sofern er hierdurch keinen wesentlichen, dauerhaften körperlichen Schaden erleidet. Sogar verstümmelnde Eingriffe wie Piercings sind schließlich erlaubt.

\section{C.1.2.3. Aufklärungspflicht}

Sofern für die in Frage stehenden Forschungszwecke nur solche Körpermaterialien verwendet werden sollen, die ohne ärztlichen Eingriff gewonnen werden können, muss der Spender nach diesseitiger Ansicht nur allgemein über den Verwendungszweck aufgeklärt werden. Wenn personenbezogene Daten gespeichert werden, muss zudem eine datenschutzrechtliche Aufklärung erfolgen. Ist indes ein ärztlicher Eingriff in den Körper des Spenders notwendig, um das Material zu gewinnen, so sind die Anforderungen an die der Einwilligung vorausgehende ärztliche Aufklärung wesentlich höher anzusetzen, als bei einem medizinisch indizierten Eingriff. Der Arzt hat den Spender vor Durchführung des Eingriffs umfassend über den Eingriff als solches, seinen Zweck, die Art und Weise der Durchführung und etwaige Gefahren bzw. Komplikationen aufzuklären und hernach die Einwilligung des Spenders in den Eingriff einzuholen. 


\section{C.1.2.4. Aufbewahrungs- und Dokumentationspflicht}

Für Proben, die ohne ärztlichen Eingriff gewonnen werden, bestehen keine besonderen Aufbewahrungs- und Dokumentationspflichten, es sei denn, dies wurde vertraglich vereinbart. Eine solche Vereinbarung kann bereits dadurch zustande kommen, dass dem Spender das Recht auf jederzeitige Vernichtung oder Herausgabe der Proben eingeräumt wird. Ist ein ärztlicher Eingriff zur Gewinnung der Proben notwendig, obliegen dem Arzt bezogen auf diesen Eingriff dieselben Dokumentationspflichten wie einem behandelnden Arzt. Allerdings hat der Arzt keine Pflicht, die Probe aufzubewahren oder deren Verbleib dauerhaft zu dokumentieren. Die Pflichten des Arztes enden, wenn er die Probe entsprechend dem sich aus Probandenvertrag und Einwilligungsklärung ergebenen Willen des Spenders an die BMB übergeben hat.

\section{C.1.2.5. Datenschutz und ärztliche Schweigepflicht}

Der entnehmende Arzt unterliegt ebenso der ärztlichen Schweigepflicht wie der behandelnde Arzt. Aus der Einwilligungserklärung und dem Probandenvertrag ergibt sich indes eine Entbindung von der ärztlichen Schweigepflicht bezogen auf diejenigen Informationen, die für das Probandenverhältnis relevant sind. Gleichwohl ist dem entnehmenden Arzt zu empfehlen, sich gezielt und ausdrücklich von der ärztlichen Schweigepflicht gegenüber der BMB entbinden zu lassen, wo dies sachlich geboten ist. Dies gilt insbesondere wegen der weithin vertretenen Auffassung, dass die Schweigepflicht des Arztes auch im Rahmen der medizinischen Forschung grundsätzlich zu beachten ist. ${ }^{118}$ Für die Schweigepflicht ist es unerheblich, ob der Arzt die in Frage stehenden Informationen aufgrund besonderen Vertrauens in Ausübung seines Arztberufes erhält; sie erfasst auch solche Patienteninformationen, von denen er auf andere Art und Weise (z. B. durch Akteneinsicht) Kenntnis erlangt. Von der ärztlichen Schweigepflicht werden nach $\int 203$ Abs. 3 S. 2 StGB auch die „berufsmäßig tätigen Gehilfen“ der Ärzte erfasst, d.h. Krankenschwestern, Arzthelferinnen, Sekretärinnen, Labormitarbeiter etc. Eine ehrenamtliche Tätigkeit reicht hierfür aus. Aus Gründen der Rechtssicherheit sollte allen diesen Mitarbeitern eine ausdrückliche und umfassende Verschwiegenheitsverpflichtungserklärung abverlangt werden.

\section{C.1.2.6. Pflicht zur Einschaltung einer Ethikkommission}

Die Pflicht zur Einschaltung einer Ethikkommission betrifft nur Ärzte, da nur diese dem Anwendungsbereich des $₫$ I5 MBO-Ä unterliegen. Sofern das Arztrecht nicht betroffen ist, weil Körpermaterialien ohne einen ärztlichen Eingriff z.B. durch einen Nicht-Arzt gewonnen werden können, besteht in der Regel keine Pflicht zur Einschaltung der Ethikkommission. Wird das Körpermaterial anders als durch einen medizinischen Eingriff durch einen Arzt ausschließlich 
für Forschungszwecke gewonnen (z.B. eine Spermaprobe), so ist die Einschaltung der Ethikkommission notwendig, wenn der Spender zumindest psychisch von der Maßnahme betroffen ist. Eine Ausnahme gilt ansonsten nur für die epidemiologische Forschung. Ist für die Probenentnahme ein medizinischer Eingriff notwendig, so liegt demgegenüber eine Forschung am Menschen im Sinne des \I5 MBO-Ä vor. In diesem Fall muss sich der Arzt nach \I5 MBO-Ä durch eine Ethikkommission beraten lassen.

\section{C.1.2.7. Nachvertragliche Aufklärungs- und Informationspflicht}

Anders als der behandelnde Arzt hat der entnehmende Arzt nach Gewinnung des Körpermaterials nicht die Pflicht, den Spender über etwaige relevante Untersuchungsergebnisse $\mathrm{zu}$ informieren, da der vorliegende atypische Arztvertrag nicht auf eine Diagnose oder Behandlung gerichtet ist. Der Arzt hat vielmehr nur die Pflicht, das Körpermaterial nach den Regeln der ärztlichen Kunst $\mathrm{zu}$ entnehmen und eine hierdurch möglicherweise entstehende Wunde entsprechend zu versorgen. Sobald diese Tätigkeit abgeschlossen und das Material zum Zwecke der weiteren Forschung der BMB zugeführt wurde, hat der Arzt seine Pflichten erfüllt. Etwas anderes gilt, wenn im Probandenvertrag eine abweichende Regelung getroffen wurde. Soweit Laufs es als „unärztlich“ ansieht, wenn der Träger einer bestimmten Krankheit, die im Rahmen eines anonymisierten Forschungsverfahren festgestellt wird, hierüber nicht aufgeklärt werden kann, ${ }^{119}$ hat er grundsätzlich recht. Dies ist aber nicht schädlich. Wenn der Arzt nur forschend an dem anonymisierten Körpermaterial tätig wird, übt er gerade keine ärztliche Tätigkeit aus. Gleichwohl sollte aus Rechtssicherheitsgründen ein entsprechend aufklärender Hinweis, besser noch der ausdrückliche Verzicht auf die Mitteilung krankheitsrelevanter Ergebnisse, in die Patienteninformation und die Einwilligungserklärung bzw. den Probandenvertrag aufgenommen werden.

\section{C.1.3. Unter welchen Umständen besteht zwischen Spender und BMB ein Arzt-Patientenverhältnis?}

Ein Arzt-Patientenverhältnis besteht zwischen Arzt und Spender, wenn ein ärztlicher Eingriff zur Gewinnung des Körpermaterials notwendig ist. Das ArztPatienten-Verhältnis besteht dabei jedoch regelmäßig nur zwischen dem tatsächlich handelnden Arzt und dem Spender. Die BMB als juristische Person ist in dieses Verhältnis regelmäßig nicht involviert. Etwas anderes gilt nur, wenn ein Krankenhaus oder eine andere medizinische Einrichtung Träger der BMB ist und ein Krankenhausvertrag besteht. Die BMB bzw. ihr Träger hätten in diesem Fall für etwaige Pflichtverletzungen des Arztes einzustehen, wenn er bei der BMB angestellt ist. 
Teil C: Relevanz von Arzt- und Arbeitsrecht

\section{C.1.4. Zusammenfassung und Empfehlung}

Das Arztrecht ist für die BMB stets dann relevant, wenn die Gewinnung der Proben nur durch einen medizinischen Eingriff möglich ist, der dem Arztvorbehalt unterliegt. Die BMB ist dann verpflichtet, einen Arzt zum Zwecke der Probengewinnung hinzuzuziehen. Ansonsten ist das Arztrecht vorrangig für den Arzt relevant, der den zur Probengewinnung notwendigen medizinischen Eingriff vornimmt. Er ist verantwortlich für die Einhaltung seiner berufsrechtlichen Pflichten. Über diese Pflichten darf er sich auch nicht hinwegsetzen, wenn er bei einer BMB angestellt oder aufgrund eines freiberuflichen Dienstvertrages für diese tätig ist. Die BMB hat dies zu akzeptieren und zu respektieren, dass der Arzt sich Weisungsfreiheit gegenüber nicht-ärztlichen Dritten vorbehalten muss.

Ein medizinischer Eingriff in den Körper eines Spenders ist nur zulässig, wenn der Spender in den Eingriff eingewilligt hat, nachdem er zuvor vollumfänglich über dessen Zweck, Umfang und Risiken sowie die anschließende Verwendung des Körpermaterials aufgeklärt worden ist. Körpermaterialien, die von einem Arzt im Rahmen eines Behandlungs- oder Diagnosevertrages entnommen worden sind, können regelmäßig nur dann für Forschungszwecke verwendet werden, wenn der Patient nach vorangegangener Aufklärung über die beabsichtigte Verwendung hierin eingewilligt hat. Eine BMB sollte daher keine Proben nutzen, für die eine entsprechende Einwilligung nicht vorliegt, da sie sich andernfalls wegen Verletzung der allgemeinen Persönlichkeitsrechte des Patienten schadenersatzpflichtig machen könnte.

\section{C.2. Die Bedeutung des Arbeitsrechts für BMB}

Das Arbeitsrecht spielt im Verhältnis zwischen dem Träger einer BMB als Arbeitgeber und den beim Träger beschäftigten Arbeitnehmern (d.h. Ärzte, Naturwissenschaftler, medizinisches und biotechnisches Hilfspersonal) eine maßgebliche Rolle. Die Begründung des Arbeitsverhältnisses richtet sich grund-

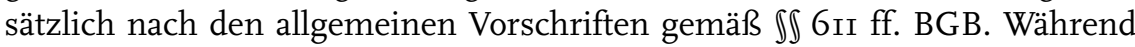
des Bestehens des Arbeitsverhältnisses finden alle einschlägigen tarifvertraglichen und gesetzlichen Regelungen, wie z.B. das BetrVerfG, das BUrlG, das AZG, das MuSchG und das TzBfG Anwendung. Bei Beendigung des Arbeitsverhältnisses findet das KSchG Anwendung, wenn der Träger der BMB eine entsprechende Betriebsgröße aufweist.

\section{C.2.1. Mögliche Organisationsformen einer BMB}

Verschiedene arbeitsrechtliche Fragestellungen hängen vom Organisationsgrad der BMB ab. So kann es z.B. für die Anwendbarkeit eines Tarifvertrages oder des KSchG relevant sein, ob eine BMB ein Betrieb, eine Betriebsabteilung oder nur ein Betriebsmittel ist. Darüber hinaus muss zwischen privater und öffentlich-rechtlicher Trägerschaft unterschieden werden. 


\section{C.2.1.1. BMB in privater Trägerschaft}

Gemäß mehrfach zitierter Definition des Nationalen Ethikrates sind Biobanken

„privat oder öffentlich unterhaltene Einrichtungen zur langfristigen Speicherung von Substanzen des menschlichen Körpers und zur Speicherung personenbezogener Daten und Informationen über den Spender dieser Körpersubstanzen, die für die Forschung eingerichtet bzw. (weiter) verwendet werden“.

Bestandteil einer BMB sind mithin Substanzen menschlicher Körper, die endgültig vom Körper ihrer Spender getrennt und nicht zur Reintegration vorgesehen sind. Andererseits gehört zu einer BMB das technische Gerät, das für eine sachgerechte Lagerung der Substanzen und zur Speicherung der zugehörigen Daten notwendig ist. Biomaterialbanken sind demnach zunächst einmal nur Lager, Sammlungen oder Archive im Besitz der Einrichtung, die sie angelegt hat, und sowohl das Biomaterial als auch das technische Gerät stellen deren Sach- bzw. Betriebsmittel dar.

Sofern die BMB als Betriebsmittel mit Personalressourcen zu einem gewissen Grad räumlich und organisatorisch abgrenzbar und selbständig ist, ihre Rolle aber nicht über eine Teilfunktion beim Erreichen des verfolgten Zweckes hinausgeht, ist sie als Unternehmens- bzw. Betriebsteil oder Abteilung einer Organisation bzw. eines Unternehmens anzusehen. Ist die BMB auf Dauer angelegt, und bildet sie zugleich eine wirtschaftliche und organisatorische Einheit mit einer Gesamtheit von Personen zur Verfolgung eines bestimmten wirtschaftlichen oder ideellen Zwecks des Unternehmers (z. B. Lagerung der Substanzen), so stellt die BMB selbst ein Unternehmen oder einen Betrieb dar. Ein Anhaltspunkt für die Abgrenzung zum Status eines Betriebsteils ist die Verselbständigung in Form einer eigenständigen Firma (z.B. ausgegliederte $\mathrm{GmbH}$ ). Betreiben mehrere selbständige Betriebe gemeinsam eine organisatorisch abgegrenzte Einrichtung unter einheitlicher Leitung liegt ein so genannter Gemeinschaftsbetrieb vor.

\section{C.2.1.2. BMB in öffentlich-rechtlicher Trägerschaft}

Im öffentlich-rechtlichen Bereich werden Biomaterialbanken vorrangig von Hochschulen, Hochschulkliniken und öffentlich-rechtlichen Krankenhäuser unterhalten. Daneben ist auch eine verselbständigte Einrichtung in Form einer juristischen Person des Privatrechts unter maßgeblicher öffentlich-rechtlicher Beteiligung denkbar. In diesem Fall würde allerdings ein eigenständiger Betrieb vorliegen, und öffentliches Dienstrecht fände nur Anwendung, wenn dies arbeitsvertraglich vereinbart wurde.

Sofern Biomaterialbanken unmittelbar von Hochschulen oder Hochschulkliniken unterhalten werden, können sie wissenschaftliche Einrichtungen, Betriebseinheiten oder Zentraleinrichtungen sein. Wissenschaftliche Einrichtungen und Betriebseinheiten sind die wohl kleinsten Teilorganisationseinheiten einer Hochschule. Sie sind regelmäßig an einen oder mehrere Fachbe- 
reiche organisatorisch angebunden. Die Errichtung einer wissenschaftlichen Einrichtung ist regelmäßig nur zulässig, wenn für bestimmte wissenschaftliche Aufgaben auf Dauer Personal- und Sachmittel zur Verfügung gestellt werden müssen. Diese Mittel stehen dann regelmäßig allen Forschern, Hochschullehrern, Studenten und Mitarbeitern zur unmittelbaren Nutzung zur Verfügung. Beispiele für wissenschaftliche Einrichtungen sind zentrale Sammlungen und Labors für Lehre und Forschung. Während die Hauptaufgabe wissenschaftlicher Einrichtungen in der wissenschaftlichen Arbeit besteht, dient die Betriebseinheit der Unterstützung solcher Arbeiten. Sie produzieren nicht unmittelbar wissenschaftliche Leistungen, sondern erbringen Dienstleistungen oder stellen Sachgüter bereit, die mittelbar der Erbringung wissenschaftlicher Leistungen dienen. Beispiele für Betriebseinheiten sind Rechenzentren und Botanische Gärten.

Entgegen früherer gesetzlicher Regelungen ( $\$ 66 \mathrm{HRG}$ ) trifft das HRG heute zwar keine verbindliche Unterscheidung zwischen wissenschaftlichen Einrichtungen und Betriebseinheiten mehr, die meisten Länder halten in ihren Landeshochschulgesetzen aber an dieser Unterscheidung fest. Als Faustregel für die Abgrenzung zwischen wissenschaftlicher Einrichtung und Betriebseinheit wird oft der Grundsatz herangezogen, dass eine wissenschaftliche Einrichtung in der Regel unter der unmittelbaren Leitung eines oder mehrerer Professoren steht, wobei die Leitung selten monokratisch ist. Selbst wenn die Leitung nur durch einen Hochschullehrer erfolgt, hat dieser häufig nach festgelegten Grundsätzen, etwa des Fachbereichs oder eines Institutsrates, zu handeln. Dem gegenüber werden Betriebseinheiten selten von einem Hochschullehrer und in der Regel monokratisch geleitet. Sofern eine BMB nur Lager- und Verwaltungsfunktionen hat, wird sie eher als Betriebseinheit anzusehen sein. Ansonsten haben BMB den Stellenwert wissenschaftlicher Einrichtungen. Unabhängig davon sind sie jedoch keine rechtlich selbständigen Einrichtungen und insofern mit Betriebsabteilungen von privaten Unternehmen zu vergleichen.

Wissenschaftliche Einrichtungen und Betriebseinheiten können auch als Zentraleinrichtungen für mehrere Hochschulen eingerichtet werden. In der Regel erfolgt dies zur Bewältigung von Daueraufgaben. Die Errichtung einer Zentraleinrichtung bedarf häufig der Zustimmung des Senates und teilweise der Genehmigung des Ministeriums. Die Leitung einer Zentraleinrichtung erfolgt z. B. durch einen Direktor. Zentraleinrichtungen sind mit Gemeinschaftsbetrieben im privatwirtschaftlichen Bereich zu vergleichen.

Wissenschaftliche Einrichtungen werden nicht selten auch als An-Institute außerhalb von Hochschulen eingerichtet. Dadurch soll die Erbringung von Dienstleistungen (z. B. Materialprüfung), die nicht zu den regelmäßigen Aufgaben von Hochschulen gehören, sowie der Austausch mit privatwirtschaftlichen Einrichtungen ermöglicht bzw. erleichtert werden. Träger eines An-Instituts ist meistens ein Dritter in der privaten Rechtsform des eingetragenen Vereins, der $\mathrm{GmbH}$ oder einer Stiftung. An-Institute sind organisatorisch regelmäßig nicht mit Hochschulen verbunden und unterliegen damit z. B. auch nicht dem jeweiligen Landeshaushaltsrecht. Das An-Insititut ist regelmäßig rechtlich selbständig und daher als Betrieb oder Unternehmen anzusehen. 


\section{C.2.2. Arbeitsrechtliche Bedeutung des Organisationsgrades einer BMB}

\section{C.2.2.1. Kündigungsschutz}

Der Organisationsgrad einer BMB ist von wesentlicher Bedeutung für die Anwendbarkeit des KSchG, da diese an den Betrieb und die Betriebsgröße geknüpft ist. Es findet in seiner seit dem or.or.2004 geltenden Fassung nur dann Anwendung, wenn in dem Betrieb mehr als Io Arbeitnehmer (bis zum 3I.I2.2003 mehr als 5 Arbeitnehmer) beschäftigt werden. Wird eine BMB als organisatorisch eigenständiger Betrieb mit maximal Io Arbeitnehmern geführt, findet das KSchG also mithin keine Anwendung. Wird die BMB indes organisatorisch als Betriebsabteilung geführt, so sind bei der Frage der Anwendbarkeit des KSchG auch die Arbeitnehmer zu berücksichtigen, die nicht unmittelbar in der BMB beschäftigt sind. Wird die BMB als Gemeinschaftsbetrieb von zwei Unternehmen geführt, die jeweils z.B. nur 8 Mitarbeiter haben, aber 3 zusätzliche Mitarbeiter im Gemeinschaftsbetrieb beschäftigen, so wird die Gesamtzahl der Mitarbeiter addiert, so dass letztlich der Schwellenwert des KSchG überschritten wird und dieses zur Anwendung kommt.

Die Sozialauswahl knüpft ebenfalls an den Betriebsbegriff an. So muss die Sozialauswahl sich auf alle vergleichbaren Mitarbeiter des Betriebes erstrecken. Auch die Möglichkeit der Weiterbeschäftigung wird zunächst im Betrieb und im Unternehmen (nur in Ausnahmefällen im Konzern) geprüft. In beiden Fällen ist mithin der Prüfungsmaßstab weiter gefasst, wenn ein gemeinsamer Betrieb vorliegt.

Während des Bestandes eines Arbeitsverhältnisses ist die Versetzung von Arbeitnehmern von einem Betriebsteil in einen anderen regelmäßig leichter als die Versetzung von einem Betrieb in einen anderen. Auch die Schließung oder Zusammenlegung von Betreibsteilen ist in der Regel einfacher, als die Schließung von Betrieben.

\section{C.2.2.2. Kollektivarbeitsrecht}

Auch das Betriebsverfassungsgesetz stellt auf den Betriebsbegriff ab. Betriebsräte können eingerichtet werden, sobald im Betrieb 5 wahlberechtigte Arbeitnehmer beschäftigt werden. Allerdings werden auch solche Betriebsteile wie selbständige Betriebe behandelt, in denen mindestens 5 Arbeitnehmer beschäftigt sind, und die räumlich weit vom Hauptbetrieb entfernt bzw. durch Aufgabenbereich und Organisation eigenständig sind. Im öffentlich-rechtlichen Bereich wird stets das BPersVG oder eines der einschlägigen Personalvertretungsgesetze der Länder Anwendung finden. Wenn eine Dienststelle nicht mindestens 5 Arbeitnehmer beschäftigt, wird sie der übergeordneten Stelle im Einvernehmen mit der Stufenvertretung einer benachbarten Dienststelle zugeordnet. Anders als eine BMB in privater Trägerschaft wird eine BMB in öffentlich-rechtlicher Trägerschaft niemals ohne Arbeitnehmer- bzw. Personalvertretung geführt werden können, was ein Hemmnis für die Flexibilität der Einrichtung darstellen kann. 
Auf BMB in öffentlich-rechtlicher Trägerschaft finden in den alten Bundesländern der BAT bzw. TVöD (einschließlich der dazu gehörenden Rationalisierungsschutzabkommen) Anwendung, in den neuen Bundesländern der BAT-O oder, sofern sich die BMB in der Trägerschaft eines Krankenhauses befindet, einer der einschlägigen Tarifverträge. Besondere Tarifverträge für BMB in privater Trägerschaft sind nicht ersichtlich. Zu beachten ist hierbei, dass eine BMB, die in privater Rechtsform von der öffentlichen Hand betrieben wird, nicht den tariflichen Vorschriften des öffentlichen Dienstes unterliegt.

\section{C.2.2.3. Individualarbeitsrecht}

Es ist davon auszugehen, dass eine BMB in privater Trägerschaft Arbeitsverträge regelmäßig wesentlich flexibler gestalten kann, als eine BMB in öffentlichrechtlicher Trägerschaft. Während eine BMB in öffentlich-rechtlicher Trägerschaft den einschlägigen tariflichen Bestimmungen unterliegt, kann eine BMB in privater Trägerschaft die Arbeitsvertragsbedingungen frei aushandeln, soweit dem nicht zwingende gesetzliche Einschränkungen entgegenstehen.

\section{C.2.2.3.1. Arbeitszeit und Urlaub}

Der gesetzliche Mindesturlaub beträgt 20 Arbeitstage im Fall der 5 Tage Woche und 24 Arbeitstage für die 6 Tage-Woche. Das sind mindestens 6 Urlaubstage weniger als im öffentlichen Dienst tarifvertraglich vorgesehen. Die werktägliche Arbeitszeit kann bis zu 48 Stunden pro Woche an 6 Arbeitstagen betragen. Die tägliche Arbeitszeit darf bis zu Io Stunden betragen. In der Privatwirtschaft sind derartige Verlängerungen der Arbeitszeit ohne Mehrvergütung als Folge der derzeitigen Arbeitsmarktbedingungen immer häufiger anzutreffen; im öffentlichen Dienst sind sie mit Ausnahme des klinischen Bereichs undenkbar. Dort darf im klinischen Bereich allerdings per Tarifvertrag auch eine noch längere tägliche Arbeitszeit vereinbart werden. Für Mitarbeiter einer BMB dürfte dies aber nur gelten, wenn die BMB an einer Klinik angesiedelt ist. Eine BMB, die auf einen selbständigen Rechtsträger ausgegliedert und als reine Lager- oder Forschungseinrichtung betrieben wird, ist nach diesseitiger Ansicht keine klinische Einrichtung und fällt daher nicht in den Anwendungsbereich der einschlägigen Tarifverträge.

\section{C.2.2.3.2. Teilzeit und Befristung}

Die Befristung von Arbeitsverhältnissen ist im Teilzeit- und Befristungsgesetz (TzBfG) geregelt. Nach 』I4 Abs. I TzBfG bedarf die Befristung eines Arbeitsverhältnisses grundsätzlich eines Sachgrundes. Nach \I4 Abs. 2 TzBfG kann aber eine Befristung ohne Sachgrund bis zu 2 Jahren erfolgen, wenn mit demselben Arbeitgeber noch kein Arbeitsverhältnis bestand. Während dieser Zeit kann das Arbeitsverhältnis maximal dreimal verlängert werden. In den ersten vier Jahren nach Gründung eines Unternehmens darf die sachgrundlose Befristung nach $\int \mathrm{I} 4 \mathrm{Abs}$. 2a TzBfG sogar bis zu 4 Jahre betragen. Die Befristung 
des Arbeitsverhältnisses von Arbeitnehmern, die älter als 58 Jahre alt sind, bedarf nach 』I4 Abs. 3 TzBfG ebenfalls keines Sachgrundes, wenn kein enger sachlicher Zusammenhang mit einem vorausgegangenen unbefristeten Arbeitsverhältnis besteht.

Für den öffentlichen Dienst finden sich abweichende Vereinbarungen in den $\iint 57$ a bis 57 f HRG und in den tarifvertraglichen Regelungen im SR 2y BAT, die auch für den neuen Tarifabschluss im öffentlichen Dienst gelten. Diese Sonderregelungen dienen einerseits der Definition, in welchen Fällen ein Sachgrund vorliegt, andererseits haben sie eine Appellfunktion. Es sollen grundsätzlich keine Zeitangestellte beschäftigt werden, sofern nicht einer der zahlreichen Ausnahmetatbestände vorliegt. Außerdem sollen befristet Beschäftigte bei der Besetzung von Dauerarbeitsplätzen bevorzugt berücksichtigt werden.

\section{C.2.2.3.3. Vergütung}

Eine BMB in privater Trägerschaft kann die Vergütung mit den Mitarbeitern weitestgehend frei aushandeln. Eine BMB in öffentlich-rechtlicher Trägerschaft ist an die tarifvertraglichen Vorgaben gebunden. Dies bedeutet nicht selten, dass insbesondere für einfachere Tätigkeiten höhere Vergütungen als in der privaten Wirtschaft gezahlt werden müssen.

\section{C.2.2.3.4. Weisungsbefugnis}

Bei einer BMB beschäftigte Ärzte sind im Kern ihrer ärztlichen Tätigkeit weisungsfrei, wobei hinsichtlich des Grades der Weisungsfreiheit zwischen Stationsärzten, Oberärzten und Chefärzten unterschieden wird. Der Stationsarzt unterliegt, soweit es um die Ausgestaltung der nichtfachlichen Rahmenbedingungen seines Arbeitsverhältnisses geht, dem Weisungsrecht seines Arbeitgebers. In fachlicher Hinsicht unterliegt er nur einem Weisungsrecht seiner ärztlichen Vorgesetzten, also von Oberarzt und Chefarzt. Für den Oberarzt gilt im Prinzip das Gleiche. Er unterliegt in fachlicher Hinsicht nur dem Weisungsrecht des Chefarztes. Der Chefarzt unterliegt regelmäßig weder in fachlicher noch in organisatorischer Hinsicht einem Weisungsrecht. Chefärzte leiten eine Abteilung oder Klinik. Sie werden regelmäßig nicht als Angestellte, sondern aufgrund eines freien Dienstvertrages nach \6II BGB beschäftigt. In diesen Verträgen werden die Rechte und Pflichten der Chefärzte individuell mit den Arbeitgebern ausgehandelt. Die genannten Regelungen beziehen sich allerdings nur auf ärztliche Tätigkeiten. In allen anderen Angelegenheiten unterliegt auch ein angestellter Arzt ebenso wie das nicht-medizinische Personal und das medizinische Hilfspersonal den Weisungen seines Arbeitgebers oder Vorgesetzen. Mit anderen Worten, ein bei einer BMB beschäftigter Arzt ist nur dann in fachlicher Hinsicht weisungsfrei, wenn er eine ärztliche Tätigkeit ausübt, was in der Regel nur im Zusammenhang mit der Entnahme des Körpermaterials der Fall sein wird. 
Teil C: Relevanz von Arzt- und Arbeitsrecht

\section{C.2.2.3.5. Leiharbeit}

Medizinisches Hilfspersonal und Ärzte können grundsätzlich auch als Leiharbeitnehmer beschäftigt werden. Ein gesetzliches Verbot besteht insoweit (auch im öffentlichen Dienst) nicht. Bestimmte Aufgaben sind allerdings aus tatsächlichen Gründen nicht der Leiharbeit zugänglich (z.B. die Position des Chef- oder Oberarztes), da Leiharbeitsverhältnisse in der Regel nur zur Deckung eines vorübergehenden Bedarfs begründet sind. Zudem kann eine Beschäftigung von Leiharbeitnehmern unter datenschutzrechtlichen Gesichtspunkten problematisch sein. Es ist daher ratsam, Leiharbeitnehmer in einer BMB durch gesonderte Erklärung zur Verschwiegenheit zu verpflichteten und ihnen gegebenenfalls keinen Zugang zu hoch sensiblen Daten zu gewähren.

\section{C.2.2.3.6. Arbeitnehmerüberlassung}

Arbeitnehmer einer BMB, einschließlich der bei ihr beschäftigten Ärzte, können grundsätzlich auch an andere Institutionen ausgeliehen werden; ein ausdrückliches gesetzliches Verbot besteht insoweit nicht. Arbeitnehmerüberlassungen sind nur dann erlaubnispflichtig, wenn sie gewerbsmäßig betrieben werden, was im vorliegenden Fall regelmäßig nicht zutreffen wird. Es ist vielmehr davon auszugehen, dass eine BMB einen Arbeitnehmer vorübergehend einem anderen Arbeitgeber (z. B. einem Projektträger) für ein bestimmtes Forschungsprojekt zur Verfügung stellen möchte. Der Arbeitnehmer bleibt in diesem Fall bei der BMB angestellt, wird aber vorübergehend in den Betrieb des anderen Arbeitgebers eingegliedert und unterliegt dessen Weisungen. Arbeitnehmerüberlassungen könnten sich allerdings immer dann als problematisch erweisen, wenn die fraglichen Mitarbeiter verbeamtet oder anderweitig als wissenschaftliche Mitarbeiter im öffentlichen Dienst beschäftigt sind. Sie können dann nicht ohne ihre Zustimmung in ein Fremdunternehmen entsandt werden.

Handelt es sich bei dem zu überlassenden Arbeitnehmer um einen Arzt, muss zusätzlich auch hinsichtlich seines personalrechtlichen Status unterschieden werden. Bei angestellten Ärzten ist eine Arbeitnehmerüberlassung weitestgehend unproblematisch, zumal wenn im Arbeitsvertrag mit der BMB ein entsprechender Versetzungsvorbehalt vorhanden ist. Behält der bisherige Arbeitgeber das Weisungsrecht, liegt ein drittbezogener Personaleinsatz aufgrund eines Werk- oder Dienstvertrages vor, der bei angestellten Ärzten ebenfalls ohne Problem möglich ist. Als dritte Variante kommt in Betracht, dass die BMB mit einem zweiten Arbeitgeber (z.B. einem Pharmaunternehmen) einen Gemeinschaftsbetrieb unterhält. Die in diesen Betrieb entsandten Arbeitnehmer sind in der Regel arbeitsvertraglich weiter an den bisherigen Arbeitgeber gebunden und unterliegen auch ausschließlich dessen Weisungsrecht, sofern nicht ausdrücklich etwas anderes vereinbart wurde. Während auch dies bei einem angestellten Arzt unproblematisch ist, sind die genannten Modelle im Falle von Chefärzten in der Regel nicht anwendbar. Ein Chefarzt ist kein Arbeitnehmer, sondern wird aufgrund eines freien Dienstverhältnisses tätig. Er kann daher nicht einseitig entsandt werden, es sei denn, dies ist im Dienstvertrag ausdrücklich vereinbart worden, oder der Chefarzt stimmt der Entsendung zu. 


\section{C.2.2.3.7. Haftung}

Die Haftung des Personals einer BMB, einschließlich angestellter Stations- oder Oberärzte, richtet sich nach den Grundsätzen des BAG zur Arbeitnehmerhaftung. Danach ist regelmäßig davon auszugehen, dass eine Haftung gegenüber dem Arbeitgeber nur für grobfahrlässiges oder vorsätzliches Fehlverhalten besteht. Die Haftung eines leitenden Chefarztes geht indes darüber hinaus. Dieser hat für etwaige, Schadenersatz begründende Behandlungsfehler in vollem Umfang selbst zu einzustehen.

\section{C.2.2.3.8. Arbeitnehmererfindungen}

Das Arbeitnehmererfindungsgesetz gilt für alle Arbeitsverhältnisse, die deutschem Recht unterliegen, und schließt in seinen Gültigkeitsbereich alle Arbeitnehmer ein $(\sqrt{ } \mathrm{I})$, auch leitende Angestellte. Mithin fallen auch Ärzte unter das Arbeitnehmererfindungsgesetz. Keine Arbeitnehmer sind freie Mitarbeiter, arbeitnehmerähnliche Personen und Organe juristischer Personen. Für sie kann aber die Gültigkeit des Arbeitnehmererfindungsgesetzes vereinbart werden. Sachlich gilt das Gesetz nach $\mathbb{2} 2$ und $\ 3$ für patent- oder gebrauchsmusterfähige Erfindungen und für technische Verbesserungsvorschläge, die vor der rechtlichen Beendigung eines Arbeitsverhältnisses fertig gestellt wurden. Es schließt Diensterfindungen ein, also Erfindungen, die entweder aus der dem Arbeitnehmer im Betrieb obliegenden Tätigkeit entstanden sind oder maßgeblich auf den Erfahrungen oder Arbeiten des Betriebes beruhen. Andere Erfindungen, selbst wenn sie durch Erfahrungen im Betrieb angeregt wurden, sind frei $\left(\mathbb{\int} 4\right)$.

Sofern eine Diensterfindung vorliegt, muss der Arbeitnehmer dies dem Arbeitgeber nach $\int 5$ unverzüglich schriftlich melden. Dies gilt auch dann, wenn er selbst bezweifelt, dass es sich um eine Diensterfindung handelt. Der Arbeitgeber kann dann entscheiden, ob er die Erfindung beschränkt oder unbeschränkt in Anspruch nimmt. Hierfür besteht nach $\int 6$ Abs. 2 eine Frist von 4 Monaten nach ordnungsgemäßer Meldung. Will der Arbeitgeber die Erfindung in Anspruch nehmen, ist er nach \I3 verpflichtet sie zum Schutzrecht anzumelden, andernfalls wird sie frei. Nimmt der Arbeitgeber die Erfindung in Anspruch, hat der Arbeitnehmer nach $\mathbb{9}$ einen Anspruch auf angemessene Vergütung. Die Vergütungshöhe ist nach Vereinbarung festzulegen. Bei unbeschränkter Inanspruchnahme ist die Vergütung gemäß \9 Abs. 2 nach der wirtschaftlichen Verwertbarkeit, den Aufgaben und der Stellung des Arbeitnehmers im Betrieb und dem Anteil des Betriebes am Zustandekommen der Erfindung zu bemessen. Eine feste Regel hierfür gibt es nicht.

Nach $\iint 42,43$ ArbNErfG müssen mittlerweile auch Hochschullehrer eine Diensterfindung unverzüglich anmelden. Dies gilt dann nicht, wenn sie die Erfindung aufgrund ihrer Forschungsfreiheit nicht offenbaren wollen. Unabhängig vom Recht der Inanspruchnahme durch den Dienstherrn hat ein Hochschullehrer das Recht, seine Erfindung zu veröffentlichen. Diese Absicht muss er aber zwei Monate vorher mitteilen, um die rechtzeitige Anmeldung der Er- 
findung zum Schutzrecht zu ermöglichen. Nach Ablauf dieser zwei Monate darf er die Veröffentlichung vornehmen, ohne dass der Dienstherr hierauf Einfluss nehmen kann. Der Erfinder erhält, auch wenn der Dienstherr die Erfindung unbeschränkt in Anspruch nimmt, in jedem Fall eine einfache Lizenz zur Benutzung der Erfindung im Rahmen seiner Hochschultätigkeit. Ferner erhält er im Falle der Verwertung durch den Dienstherrn eine Pauschale von $30 \%$ der hierdurch erzielten Einnahmen.

Hat ein Arbeitnehmer eine freie Erfindung getätigt, d.h. eine Erfindung, die nicht auf der betrieblichen Tätigkeit oder den betrieblichen Erfahrungen beruht, hat er dies nach $\ 18$ Abs. I ebenfalls unverzüglich schriftlich anzuzeigen und dem Arbeitgeber nach $\$ I9 ein nicht ausschließliches Nutzungsrecht zu angemessenen Bedingungen anzubieten. Dies gilt nach $\int$ I8 Abs. 3 nicht, wenn die Erfindung offensichtlich nicht im Unternehmen verwendbar ist.

Aus den vorangegangenen Ausführungen ergibt sich, dass es unter dem Gesichtspunkt der Arbeitnehmererfindung von besonderer Bedeutung ist, in welcher Trägerschaft (öffentlich-rechtlich oder privat) sich eine BMB befindet und welche Personen mit der Leitung der BMB betraut werden. Wird eine BMB von einem Hochschullehrer in Nebentätigkeit als Geschäftsführer geleitet, wird es ohne entsprechende Vereinbarung kaum möglich sein, diesen zu veranlassen, die Rechte an etwaigen von ihm im Rahmen eines Forschungsauftrages der BMB gemachten Erfindungen der BMB zu überlassen. Es ist daher zu empfehlen, die organschaftliche Leitung einer BMB einer rein kaufmännisch tätigen, nicht forschenden Person zu übertragen und in den Verträgen mit sämtlichen Forschern sicher zu stellen, dass die Verwertungsrechte an Erfindungen weitestgehend bei der BMB verbleiben. 